\title{
LnCRNA MALAT1 silencing protects against cerebral ischemia-reperfusion injury through miR-145 to regulate AQP4
}

Hongwei Wang ${ }^{1 \dagger}$, Xiaoxiao Zheng ${ }^{2,3 \dagger}$, Jing Jin ${ }^{4 \dagger}$, Li Zheng ${ }^{2,3}$, Ting Guan ${ }^{1}$, Yangfan Huo ${ }^{1}$, Shufen Xie', Ying $\mathrm{Wu}^{2,3^{*}}$ and Wei Chen ${ }^{2,3^{*}}$

\begin{abstract}
Background: The present study aimed to verify whether long noncoding RNA (InCRNA) MALAT1 is involved in brain tissue damage induced by ischemia-reperfusion injury, and to explore the mechanism by which MALAT1 regulates aquaporin 4 (AQP4).

Methods: In this study, we established glucose deprivation (OGD)/reoxygenation (RX) astrocyte cell model and middle cerebral artery occlusion (MCAO)/reperfusion mouse model in vitro and in vivo. Then cell counting kit-8 assay, flow cytometry analysis, Triphenyltetrazolium chloride (TTC) staining, and western blotting were used to determine cell viability, cell apoptosis, cerebral infarction volume, and the abundance of AQP4, respectively.

Results: We found that the level of MALAT1 was significantly upregulated in both the MCAO/reperfusion model and OGD/RX model. Knockdown of MALAT1 increased cell viability and reduced cell apoptosis in MA-C cells, while an AQP4 siRNA combined with a siRNA targeting MALAT1 could not enhance this effect. Further experiments showed that MALAT1 positively regulated AQP4 expression via miR-145. The MALAT1 siRNA did not alleviate the exacerbation of damage after miR-145 inhibitor action. However, an miR-145 inhibitor reversed the protection effects of MALAT1, indicating that MALAT1 silencing protects against cerebral ischemia-reperfusion injury through miR-145. TTC staining showed that the infracted area of whole brain was significantly attenuated in treated with sh-MALAT1 group in vivo.

Conclusion: Taken together, our study confirmed that MALAT1 promotes cerebral ischemia-reperfusion injury by affecting AQP4 expression through competitively binding miR-145, indicating that MALAT1 might be a new therapeutic target for treatment cerebral ischemic stroke.
\end{abstract}

Keywords: MALAT1, miR-145, Cerebral ischemia-reperfusion injury, AQP4

\footnotetext{
* Correspondence: wei_chen@zju.edu.cn; wuying@sibs.ac.cn

${ }^{\dagger}$ Hongwei Wang, Xiaoxiao Zheng and Jing Jin contributed equally to this work.

${ }^{2}$ Cancer Institute of Integrated traditional Chinese and Western Medicine, Zhejiang Academy of Traditional Chinese Medicine, Tongde hospital of Zhejiang Province, NO.234, Gucui Road, Hangzhou 310012, Zhejiang, China Full list of author information is available at the end of the article
} 


\section{Background}

Stroke is one of the most common causes of long-term severe disability and death worldwide [1, 2]. Approximately $80-85 \%$ of stroke cases are induced by cerebral ischemia, which is usually caused by embolism or thromboembolism occlusion of the major cerebral aorta [3]. Interventions require recovery of blood flow, resulting in reperfusion injury. Cerebral ischemia-reperfusion (CIR) injury is a pathological process in which nerve damage caused by ischemia and hypoxia is further aggravated following the short-term recovery of blood perfusion [4]. A growing body of evidence suggests that ischemia often involves a range of neurological events, such as, hypoxia, oxidative stress, and an inflammatory response [5], which ultimately lead to acute necrosis, apoptosis, and autophagy in the ischemic brain [6]. Currently, tissue plasminogen activator (tPA) is the only effective method to treat CIR injury [7]. Therefore, it is necessary and urgent to identify novel and effective therapeutic targets, and the underlying molecular mechanism of cerebral ischemia for patients with stroke.

Long noncoding RNAs (lncRNAs) are non-protein coding RNAs of more than 200 nucleotides in length, which participate in various biological processes, such as cell apoptosis, differentiation, angiogenesis, and proliferation [8-10]. Furthermore, IncRNAs also regulate gene expression [11] and are closely associated with various neurological diseases including ischemia stroke [12]. Metastasis-associated lung adenocarcinoma transcript 1 (MALAT1), also called non-coding nuclear-enriched abundant transcript 2 (NEAT2), was originally identified as being associated with lung cancer metastasis [13, 14]. Extensive evidence revealed that the level of MALAT1 is upregulated in many cancers and is also associated with tumor initiation, progression, and recurrence [15-17]. MALAT1 also regulates endothelial cell function and vessel growth [18]. More interestingly, Zhang et al. reported that MALAT1 was highly expressed during in vitro mimicking of ischemic stroke conditions [19]. A growing number of studies have demonstrated that MALAT1 is associated with ischemic stroke, and could reduce the number of apoptotic neuronal cells, and inhibit autophagy by regulating microRNA miR-30 in cerebral ischemic stroke [20, 21]. Furthermore, upregulation of MALAT1 could reduce the protective role of fentanyl in ischemia/reperfusion (I/R) injury by regulating the miR-145/BCL2 interacting protein 3 (BNIP3) pathway [22]. Our previous study demonstrated that overexpression of miR-145 could ameliorate astrocyte cell injury by downregulating aquaporin 4 (AQP4) expression in cerebral ischemic stroke [23]. In the present study, we employed a glucose deprivation (OGD)/reoxygenation (RX) astrocyte cell model and middle cerebral artery occlusion (MCAO)/reperfusion mouse model for in vitro and in vivo study, respectively. Then we investigated the role of $M A L A T 1$ in cerebral I/R injury, and revealed its possible underlying mechanism.

\section{Methods \\ Animals}

Six-week-old male C57BL/6 J mice (20-25 g) were purchased from the Experimental Animal Center of Zhejiang University School of Medicine. All mice were housed in an environmentally controlled room under a $12 \mathrm{~h}$ light/dark cycle with ad libitum access to food and water. All animal experiments were approved by the First Affiliated Zhejiang Hospital, Zhejiang University of Medical Ethics Committee and the Medical Faculty Ethics Committee of the First Affiliated Zhejiang Hospital, Zhejiang University in accordance with the National Institutes of Health Guide for Care and Use of Laboratory Animals (NIH Publications, No. 8023, revised 1978).

\section{Primary astrocyte culture}

Primary astrocytes were prepared from a post-natal day (PND) 7 cerebellum from C57BL/6 J mice, and the protocol used was described previously [24]. Briefly, the tissue was incubated for $15 \mathrm{~min}$ in $0.065 \%$ trypsin at $37^{\circ} \mathrm{C}$ and then centrifuged $1500 \mathrm{~g}$ for $5 \mathrm{~min}$. The cell pellet was treated with $0.004 \%$ DNase I for $7 \mathrm{~min}$ at $37^{\circ} \mathrm{C}$. After centrifugation $1500 \mathrm{~g}$ for $5 \mathrm{~min}$, the cell pellet was gently resuspended in a small volume of tissue growth medium (Dulbecco's modified Eagle's medium containing $10 \% \mathrm{FBS}$ ) and plated in the same medium at a density of $2 \times 10^{5}$ cells $/ \mathrm{cm}^{2}$ in $35-\mathrm{mm}$ Corning culture dishes precoated with $100 \mu \mathrm{g} / \mathrm{ml}$ poly-D-lysine. The cells were cultured in poly-L-lysine-coated $35-\mathrm{mm}$ dishes with DMEM containing $10 \% \mathrm{FBS}$ at $37^{\circ} \mathrm{C}$ in $5 \% \mathrm{CO}_{2}$ in a humidified.

\section{Astrocyte cell OGD/RX model}

The astrocyte cell OGD/RX model was established in accordance with the methods as previously described [25]. Briefly, the cells were transferred to glucose-free DMEM and cultivated in a humidified incubator with 95\% $\mathrm{N}_{2}$ and $5 \% \mathrm{O}_{2}$ at $37{ }^{\circ} \mathrm{C}$ for $6 \mathrm{~h}$. For reperfusion, the medium was replaced with high-glucose DMEM and incubated in a normoxic incubator for an additional $24 \mathrm{~h}$ or $48 \mathrm{~h}$.

\section{Middle cerebral artery occlusion (MCAO)/reperfusion model}

The Middle Cerebral Artery Occlusion (MCAO) procedure was described in our previous study [26]. In brief, mice were anesthetized with $4 \%$ chloral hydrate (Sigma, St. Louis, MO, USA) and a 6-0 silicone-coated nylon monofilament (Doccol Corp., Redlands, CA, USA) was inserted into the left common carotid artery to occlude the MCA origin. After $1 \mathrm{~h}$, the suture was removed. The 
blank groups prepared the filaments and inserted into the left common carotid artery. The mice were then anesthetized and decapitated to obtain the brain. C57BL/6 J mice were randomly grouped as follows $(n=$ 5 for each group): NC group (Threading without occlusion, followed by persistent perfusion), MCAO $24 \mathrm{~h}(1 \mathrm{~h}$ of ischemia and $24 \mathrm{~h}$ of reperfusion), MCAO $48 \mathrm{~h}$ ( $1 \mathrm{~h}$ of ischemia and $48 \mathrm{~h}$ of reperfusion), shMALAT1 (short hairpin RNA targeting MALAT1) + MCAO $48 \mathrm{~h}$ (0.5 $\mathrm{mg} / \mathrm{kg}$ MALAT1 shRNA, via intracerebroventricular injection before MCAO). MALAT1 shRNA or sh-NC was injected into the right cerebral ventricle of mice. One day post-injection, MCAO operation was established.

\section{Measurement of the cerebral infarction area}

Triphenyltetrazolium chloride (TTC) staining was used to determine cerebral infarction area. After $24 \mathrm{~h}$ of reperfusion, $1 \mathrm{~mm}$-thick coronal sections of the brain were immersed in a $2 \%$ TTC solution at $37^{\circ} \mathrm{C}$ for $30 \mathrm{~min}$. The total area of each brain section and the infarcted region were quantified using Leica image software DMI6000B (Leica Microsystems, Wetzlar, Germany). The infarct volume was determined as follows: (infarcted area /total brain area) $\times 100 \%$.

\section{siRNA transfection}

The miR-145 mimic, miR-145 inhibitor, or MALAT1 siRNA were synthesized by GenePharma (Shanghai, China). MA-C cells, which were obtained from ATCC (Manassas, VA, USA), were transfected using Lipofectamine 2000 (Invitrogen; Thermo Fisher Scientific, Waltham, MA, USA) according to the manufactures' instructions. The oligonucleotide and MALAT1 siRNA sequences used were as follows:

hsa-miR-145 mimics:

Forward 5'- GUCCAGUUUUCCCAGGAAUCCCU - 3'

Reverse 5' - GGAUUCCUGGGAAAACUGGACUU - 3'

hsa-miR-145 inhibitor:

5' - AGGGAUUCCUGGGAAAACUGGAC -3'

MALAT1 SiRNA:

MALAT1-1-Homo-209:

Forward 5' - GGUGGUGGUAUUUAGAUAATT - 3'

Reverse 5' - UUAUCUAAAUACCACCACCTT -3';

MALAT1-2-Homo-1790:

Forward 5' - GCGUCAUUUAAAGCCUAGUTT - 3';

Reverse 5' - ACUAGGCUUUAAAUGACGCTT -3';

MALAT1-3-Homo-4443:

Forward 5' - GGGCUGACAUUAACUACAATT - 3'

Reverse 5' - UUGUAGUUAAUGUCAGCCCTT - 3'.

\section{Western blot analysis}

Protein samples $(40 \mu \mathrm{g} / \mathrm{lane})$ were separated using $10 \%$ SDS-PAGE, and then transferred to a polyvinylidene difluoride (PVDF) membrane (Millipore, Billerica, MA,
USA). After blocking with Tris-buffered saline (TBS) and $0.1 \%$ Tween-20 (TBS/T) containing 5\% bovine serum albumin (BSA), the membranes were incubated with primary antibodies against AQP4 (Abcam, Cambridge, MA USA) diluted 1:1000 in TBS/T overnight at $4{ }^{\circ} \mathrm{C}$. The membranes were washed and incubated with a horseradish peroxidase-labeled secondary antibody diluted 1:2000 at room temperature for $2 \mathrm{~h}$. Subsequently, the protein bands were assessed using chemiluminescence (GE Healthcare, Piscataway, NJ, USA). The immunoreactive protein bands were quantified using Image Lab 5.0 (Bio-Rad, Hercules, CA, USA) and glyceraldehyde-3-phosphate dehydrogenase (GAPDH) was used as an internal control.

\section{Quantitative real-time reverse transcription PCR (qPCR)}

Total RNA was isolated using the TRIzol reagent (Invitrogen; Thermo Fisher Scientific, Inc.). cDNA was synthesized using a PrimeScript RT Reagent Kit (Takara, Shiga, Japan). After the RT reaction, $1 \mu \mathrm{l}$ of $\mathrm{cDNA}$ was used for subsequent qPCR with SYBR Green dye (Takara) using a 7500 Real-Time PCR System (Applied Biosystems; Thermo Fisher Scientific, Inc.). The PCR conditions were as follows: 40 cycles of $95^{\circ} \mathrm{C}$ for $30 \mathrm{~s}$, $60{ }^{\circ} \mathrm{C}$ for $34 \mathrm{~s}$, and $72{ }^{\circ} \mathrm{C}$ for $30 \mathrm{~s}$. U6 and $\beta$-actin were used as internal controls, and the relative expression levels were calculated using the $2^{-\Delta \Delta \mathrm{Ct}}$ method [27]. All reactions were performed in triplicate. The primer sequences were as follows:

\section{MALAT1:}

Forward 5' - TGTGACGCGACTGGAGTATG - 3'

Reverse 5' - CAAAGGGACTCGGCTCCAAT -3';

AQP4:

Forward 5'-GACAGACCCACAGCAAGG-3'

Reverse 5' - GCAAAGGGAGATGAGAACC-3'; miR-145:

5'-GTCCAGTTTTCCCAGGAATCCCT-3'; U6:

Forward 5' -CTCGCTTCGGCAGCACA-3', Reverse 5'-AACGCTTCACGAATTTGCGT-3'; $\beta$-Actin:

Forward 5' - GACTTAGTTGCGTTACACCCTT-3', Reverse 5' - TTTTGACCTTGCCACTTCCA-3'

\section{Cell Calcein-AM/PI staining assay}

Cell viability was measured using a calceinacetoxymethyl (AM)/propidium iodide (PI) double staining kit (Dojindo, Kumamoto, Japan). In brief, cells were fixed with $70 \%$ ethanol for $15 \mathrm{~min}$. They were then stained with calcein-AM solution $(10 \mu \mathrm{mol} / \mathrm{l})$ for $15 \mathrm{~min}$. They were then washed with phosphate-buffered saline (PBS) buffer and stained with PI $(10 \mu \mathrm{mol} / \mathrm{l})$ for $15 \mathrm{~min}$. The optical density at $490 \mathrm{~nm}$ and $535 \mathrm{~nm}$ were 
determined to determine healthy cells and dead cells, respectively.

\section{Lactate dehydrogenase (LDH) analysis}

The level of lactate dehydrogenase (LDH) was determined using a commercial Cytotoxicity LDH Assay KitWST according to the manufacturer's instructions (Dojindo, Kumamoto, Japan). In brief, MA-C cells were cultured in 6-well plates, following transfection and OGD/RX treatment, then collected, re-suspended, couted and seeded in a 96-well plate at $37{ }^{\circ} \mathrm{C}$ in $5 \% \mathrm{CO}_{2}$. Add $20 \mu \mathrm{l}$ of the Lysis Buffer to each well of the high control. Incubate the plate at $37^{\circ} \mathrm{C}$ for $30 \mathrm{~min}$ in a $\mathrm{CO}_{2}$ incubator. $100 \mu \mathrm{l}$ working solution was added to each well. Protect the plate from light and incubate it at room temperature for $30 \mathrm{~min}$. At last, $50 \mu \mathrm{l}$ stop solution was added to each well. The level of LDH was measured the absorbance at $490 \mathrm{~nm}$ by a microplate reader.

\section{Bederson score}

Normal mice exhibited a head lift and the two front paws extended to the tabletop when the mouse tail was raised to $5 \mathrm{~cm}$ above the table top. The brain injury mice displayed buckling of the contralateral forelimbs. The postural changes from mild flexion, elbow extension, shoulder abduction, severe wrist and elbow flexion, and shoulder internal rotation abduction were observed. The Bederson score was divided into four grades according to the posture reflex and shoulder lateral thrust test results of the mice. 0 point: no neurological deficit symptoms were observed; 1 point: buckling of the contralateral forelimbs of the infarct hemisphere when the tail was suspended, but not with other abnormalities; 2 points: when the tail is suspended, the contralateral forelimb of the infarct hemisphere is flexed, and the opposite side of the infarct hemisphere is pushed by hand, and its resistance is reduced, but it does not rotate when freely moving; 3 points: the same behavior with 2 points and when it is free to move, turn to the side of the squat.

\section{TdT-mediated biotin-16-dUTP nick-end labeling (TUNEL)} assay

A TUNEL Apoptosis Assay kit (Roche, Basel, Switzerland) was used to detect the apoptotic cells according to manufactures' instructions. Briefly, paraffinembedded sections were deparaffinized and hydrated in a graded ethanol series and then digested with trypsin for $40 \mathrm{~min}$ at room temperature. The tissue sections were then incubated with TUNEL reaction buffer in a $37^{\circ} \mathrm{C}$ humidified atmosphere for $60 \mathrm{~min}$, and then washed with PBS. TUNEL-positive cells and normal cells in each group were counted under a light microscope at 200× magnification (Olympus, Tokyo, Japan).

\section{Flow cytometry analysis}

The number of apoptotic cells in the different treatment groups was examined using Flow cytometry analysis. The cells were stained with Annexin V/fluorescein isothiocyanate (FITC) kit (BD Biosciences, Franklin Lakes, NJ, USA) according to the manufacturer's instructions. Briefly, MA-C cells were cultured in 6-well plates, following OGD/RX treatment and transfection. The cells were collected, $100 \mu \mathrm{l}$ Binding Buffer and $5 \mu \mathrm{l}$ fluorescein isothiocyanate (FITC)-labeled Annexin V $(20 \mu \mathrm{g} / \mathrm{ml})$ were added and incubated in the dark at room temperature for $15 \mathrm{~min}$. Then, $5 \mu \mathrm{l}$ propidium iodide (PI; $50 \mu \mathrm{g} / \mathrm{ml}$ ) was added and incubated in the dark for 5 min. Thereafter, $400 \mu \mathrm{l}$ of Binding Buffer was added and immediately subjected to FACScan for quantitative detection by flow cytometry (within $1 \mathrm{~h}$ ).

\section{Immunofluorescence}

Astrocyte cells were identified using immunofluorescence. Cells were washed with cold PBS, fixed in 4\% paraformaldehyde for $15 \mathrm{~min}$, blocked with $5 \%$ BSA at $37^{\circ} \mathrm{C}$ for $30 \mathrm{~min}$, and incubated with anti-glial fibrillary acidic protein (GFAP) antibodies (1:100; Abcam) overnight at $4{ }^{\circ} \mathrm{C}$. The cells were washed and incubated with secondary antibodies $\left(1: 100\right.$; Abcam) for $2 \mathrm{~h}$ at $37^{\circ} \mathrm{C}$. Nuclei were stained using 2-(4-amidinophenyl)- $1 \mathrm{H}$-indole-6-carboxamidine (DAPI; (Sigma) for $2 \mathrm{~min}$ at $37^{\circ} \mathrm{C}$. The cells were washed and observed under an inverted fluorescence microscope (Olympus, Tokyo, Japan).

\section{Statistical analysis}

All experimental values are expressed as the mean \pm SD . We used GraphPad Prism 6.0 software (GraphPad Software, San Diego, CA, USA) to perform the statistical analysis. Statistical analysis was performed using a $t$-test for the comparison of two conditions. A one-way analysis of variance (ANOVA) with a Bonferroni post-test was used for multiple comparisons. A $P$ value of $<0.05$ was considered statistically significant.

\section{Results}

Inhibition of MALAT1 could protect against astrocyte cell injury caused by ischemia- reperfusion

To search appropriate LncRNAs, we used qPCR to detect the level of lncRNAs under oxygen and glucose deprivation (OGD)/reoxygenation (RX) or Mock condition, which showed that MALAT1 was significantly upregulated under OGD/RX conditions (Fig. 1a). We established MCAO mouse model and OGD/RX cell model and then we examined the level of MALAT1 in vivo and in vitro. We found that the expression of MALAT1 was upregulated in MCAO mice at 24 and 48 $\mathrm{h}$ compared with sham group and control group (Fig. 1b). MALAT1 expression was also significantly increased 


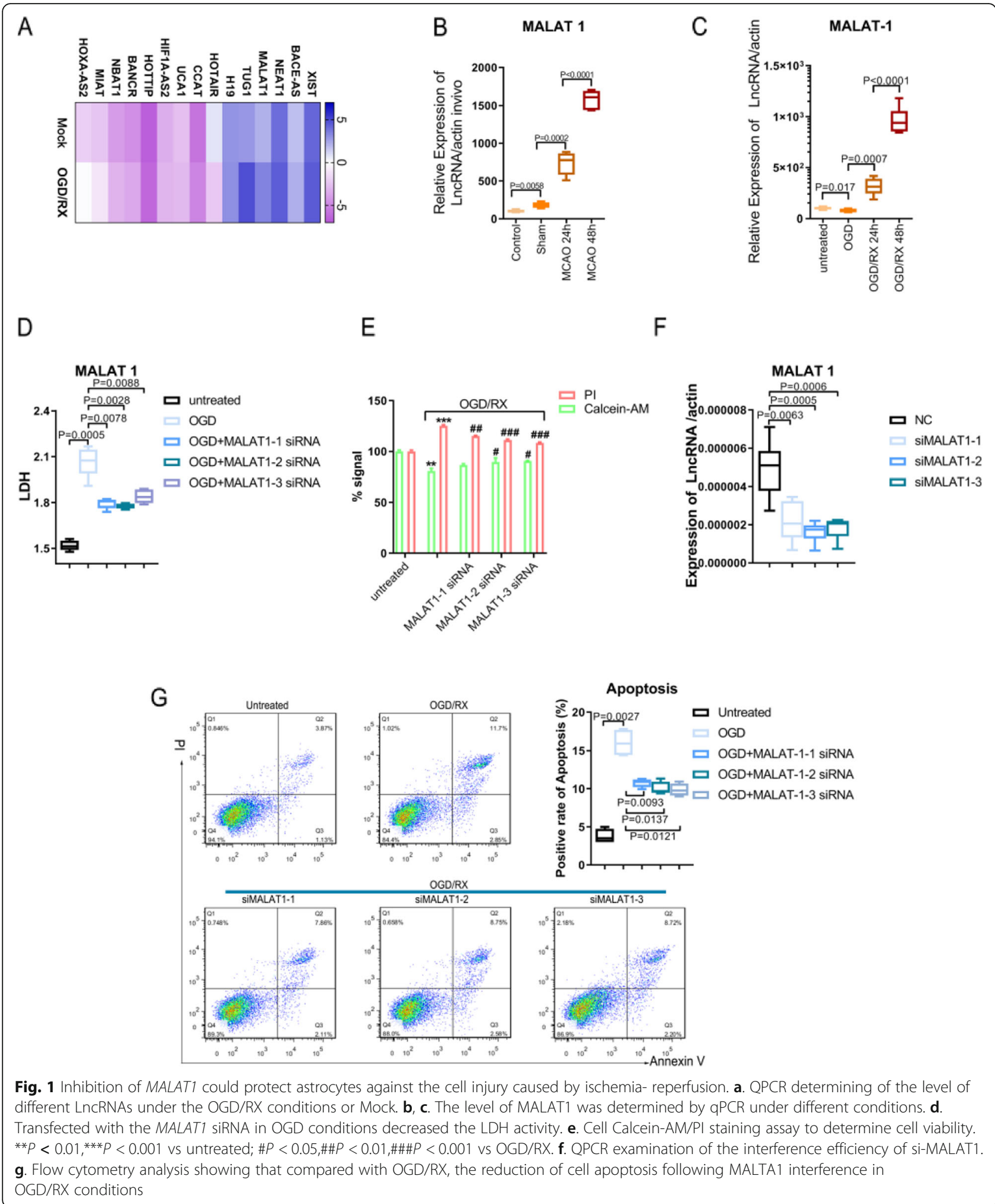

with OGD/RX for $24 \mathrm{~h}$ and $48 \mathrm{~h}$ in MA-C cells compared to the OGD only group (Fig. 1c). To observe the effect of MALAT1 on MA-C cells, a LDH assay showed that knockdown of MALAT1 could attenuate the increase in OGD-induced LDH release from cells (Fig. 1d). Cell calcein-AM/PI staining experiment was used to determine the percentage of live cells in which calcein-AM stains live cells and PI stains dead cells. The 
results showed that the percentage of live cells was significantly increased in cells treated with the MALAT1 siRNA under OGD/RX conditions (Fig. 1e). The transfection efficiency of MALAT1 siRNA was determined using qPCR (Fig. 1f). Furthermore, flow cytometry was used to determine apoptosis cells, and we found that the number of apoptotic cells was significantly reduced after lncRNA MALAT1 siRNA intervention (Fig. 1g).

\section{AQP4 mediates the effect of MALAT1 on the damage to astrocyte cells}

Our research team has been studying the role of AQP4 at the early stages of stroke brain tissue damage [23]. We also revealed that AQP4 in astrocytes may promote ischemia reperfusion injury (Figure S1). Glial fibrillary acidic protein (GFAP), the hallmark intermediate filament protein in astrocytes, a main type of glial cells in the central nervous system (CNS). GFAP immunofluorescence staining was used for identifying primary astrocytes purity (Figure S1 A). Western Blot and qPCR showed that AQP4 expression is significantly increased after OGD/RX treatment (Figure S1 B-C). To investigate the physiological roles of AQP4 in astrocytes, cells were transfected with AQP4 siRNA to knockdown AQP4 levels (Figure S1 D). Calcein-AM/PI staining were performed to examine the effect of AQP4 on cell viability of astrocytes upon OGD/RX treatment. Intriguingly, knockdown of AQP4 significantly heightened cell viability against OGD/RX treatment (Figure S1 B). Additionally, reduced AQP4 levels resulted in a decrease of cytotoxicity by LDH assay(Figure S1 E). Consistently, reduced apoptosis was determined by flow cytometric analysis with AnnexinV/PI staining in astrocytes with AQP4 siRNA upon OGD/RX treatment (Figure S1 F). As a result, we found that IncRNA MALAT1 could regulate the expression of AQP4, and AQP4 mediates the damage of MALAT1 on MA-C cells. Considering the important role of AQP4 in stroke reperfusion injury, we speculated whether LncRNAs play an important role in regulating AQP4. The level of AQP4 was detected before and after MALAT1 siRNA interference, and the results showed that the level of AQP4 was reduced after knockdown of MALAT1 (Fig. 2a). After AQP4 was successfully knocked down in MA-C cells using an siRNA, the OGD/RX model was established, and the model cells treated the MALAT1 siRNA, or not treated to observe whether the cells were damaged in the presence or absence of MALAT1. The efficiency of AQP4 siRNA silencing was determined using western blotting (Fig. 2b). The LDH and CCK-8 results showed that after AQP4 silencing, cell damage was alleviated, while combined with MALAT1 siRNA, we found that MALAT1 siRNA did not change the effect of AQP4 on the reduction of brain injury after OGD-RX induction (Fig. 2c, d). In addition, to verify whether MALAT1 play a protective effect through regulating AQP4 after OGD/RX, MA-C cells were transfected with AQP4 plasmid to overexpress AQP4 (Figure S2 C), along with or without MALAT1 siRNA upon OGD/RX treatment, respectively. Impressively, the damage effects of OGD/RX on cell viability and cytotoxicity were robustly amplified by the transfection of AQP4 plasmid, while the protective effects of siMALAT1 disappeared when cells were co-transfected with AQP4 plasmid and MALAT1 siRNA (Figure S2 AB). These findings demonstrated that MALAT1 promoted astrocytes damage through AQP4 upon OGD/RX treatment. Flow cytometry analysis showed that after $\mathrm{AQP4}$ silencing, MALAT1 did not further reduce apoptosis, again demonstrated that MALAT1 acted through AQP4 (Fig. 2e). These results demonstrated that lncRNA $M A L A T 1$ could regulate the expression of AQP4, and that AQP4 mediates the effects of MALAT1 on the damage to astrocytes.

\section{MALAT1 positively modulates AQP4 through miR-145 expression}

LncRNAs participate in the regulation of biological activity by acting as competing endogenous RNAs (ceRNAs) for miRNAs [28]. To explore the underlying mechanism of MALAT1 regulating AQP4 in cerebral ischemia-reperfusion injury, we used starBase (http:// starbase.sysu.edu.cn/) to screen miRNAs that have complementary base pairing with MALAT1. Interestingly, miR-145 was identified as a potential target of MALAT1 (Fig. 3a). QPCR detected the changes in miR-145 expression with or without MALAT1 siRNA (siMALAT1-1, siMALAT1-2 and siMALAT1-3) transfection, showing that the level of miR-145 was significantly upregulated following knockdown of MALAT1 (Fig. 3b). Bioinformatics analysis using Targetscan (http://www.targetscan. org/) indicated that miR-145 binds to the 3' UTR of the AQP4 mRNA. An miR-145 mimic significantly downregulated AQP4 levels, while an miR-145 inhibitor significantly upregulated AQP4 levels in the astrocytes. Furthermore, in the OGD/RX model, the miR-145 mimic could downregulate the increase of AQP4 caused by OGD/RX, while the miR-145 inhibitor could upregulate AQP4 level (Fig. 3c-d), which was consistent with the results of our previous study [23].

\section{MiR-145 mediates the effect of MALAT1 on damage to astrocyte cells}

To further verify whether miR-145 was a target of MALATA1 mediating its regulation of AQP4 on OGD/ RX damage to astrocyte cells, MA-C cells were pretreated with the miR-145 inhibitor along with or without the MALATA1 siRNA upon OGD/RX treatment, cell viability, cell apoptosis and cytotoxicity were detected 


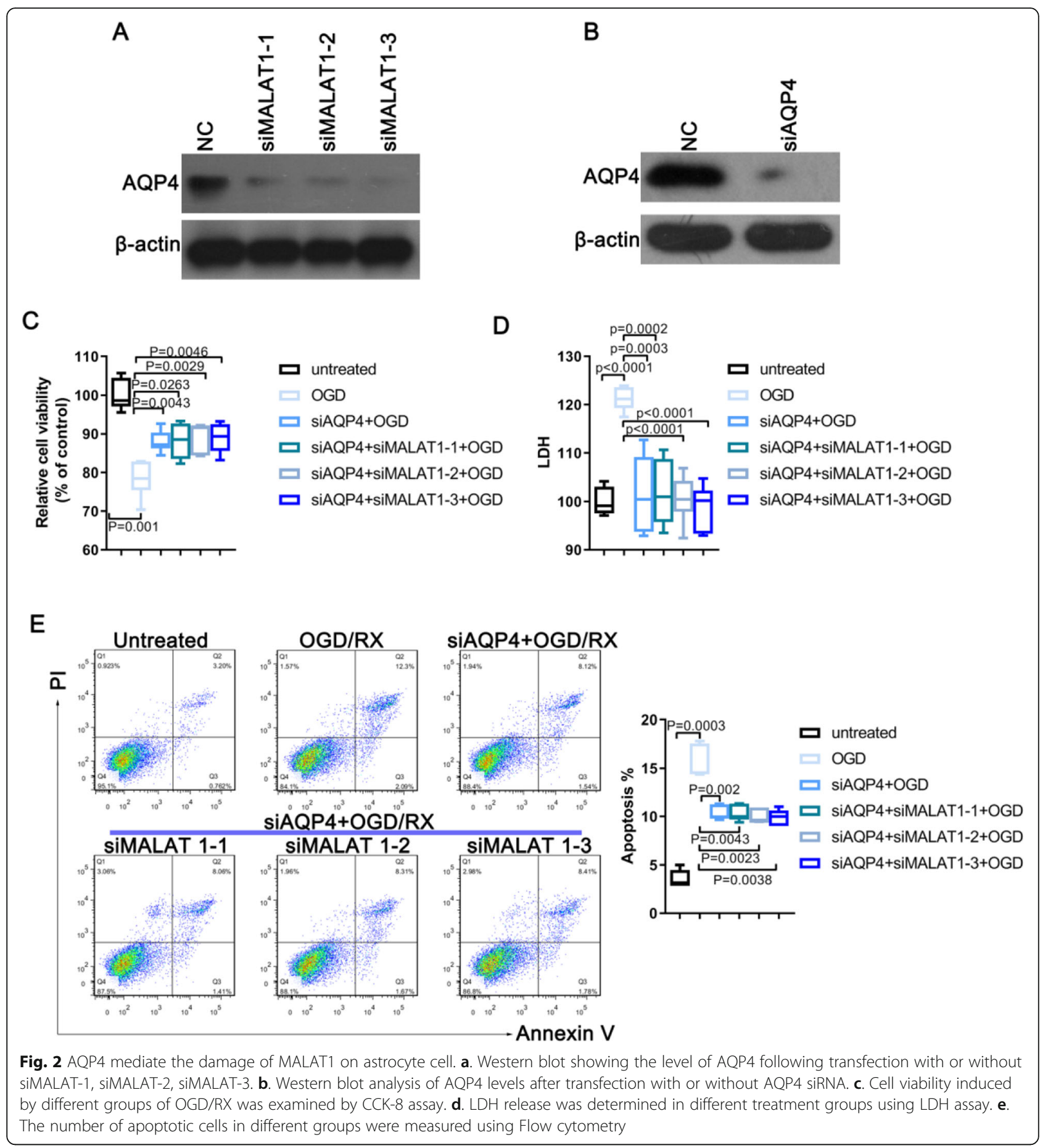

via Calcein-AM/PI staining, flow cytometric analysis and LDH assay, respectively. Flow cytometry detection of apoptotic cells revealed that after treatment with the miR-145 inhibitor, the number of apoptotic cells increased, while treatment with the MALAT1 siRNA did not influence the effect of the miR-145 inhibitor (Fig. 4a-b). Furthermore, the percentage of dead cells stained by PI and cytotoxicity LDH release increased significantly by treatment with the miR-145 inhibitor under OGD/RX conditions; however, knockdown of MALAT1 did not alleviate the astrocytes injury (Fig. 4cd). Next, we found that MALAT1 siRNA interference could reduce cell apoptosis and cytotoxicity LDH release which were induced by OGD/RX treatment. Although knockdown of MALAT1 could protect MA-C cells from OGD/RX damage, miR-145 inhibitor could reverse the 


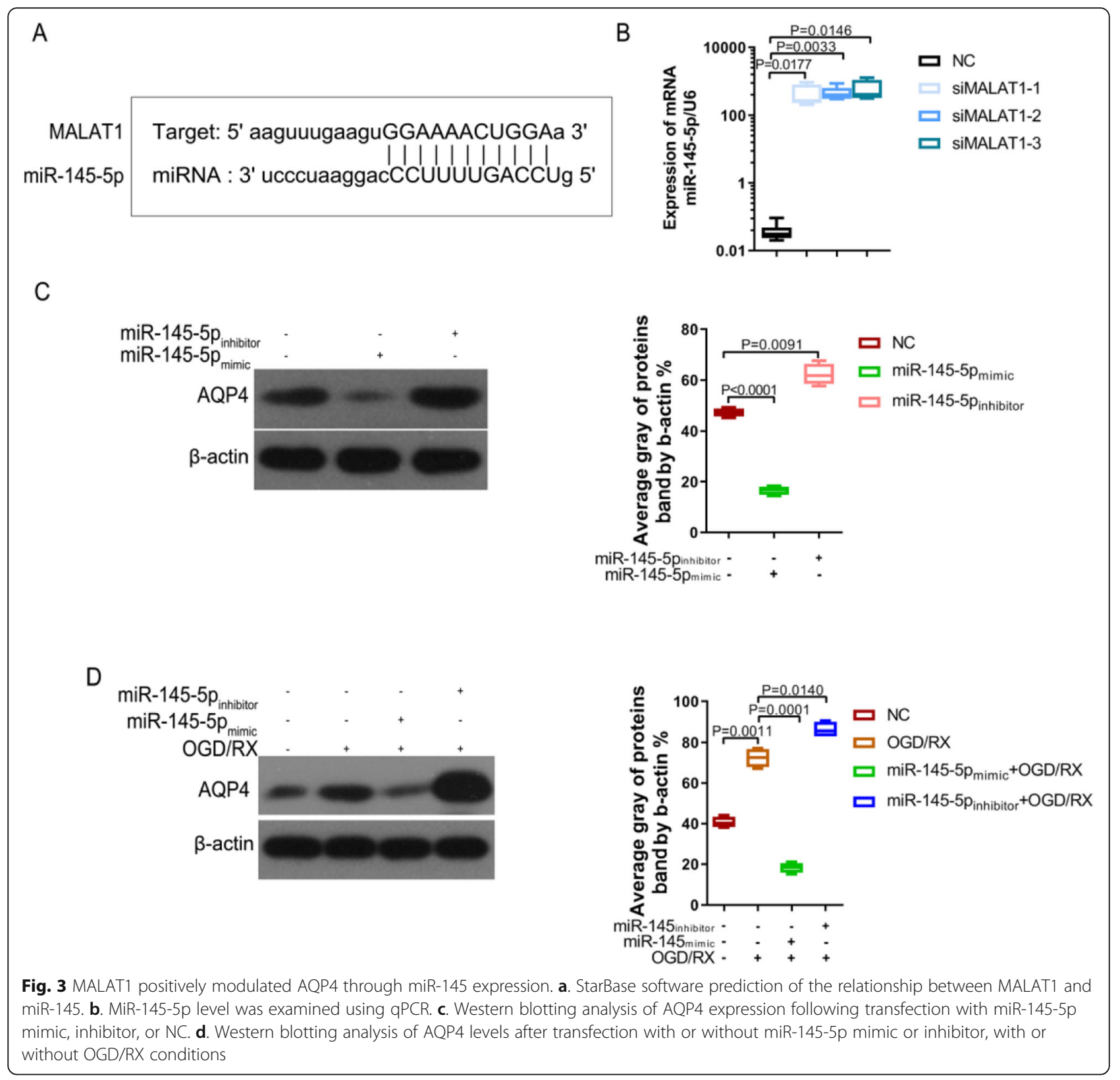

influence of MALAT1 siRNA (Fig. 4e-g). These results demonstrated that the effects of the MALAT1 siRNA act via upregulating miR-145levels.

MALAT1 is involved in the regulation of stroke injury by upregulating miR-145 in vivo

In vivo experiments confirmed that lncRNA MALAT1 was involved in the regulation of stroke damage. The brain infarct area was determined via a 2,3,5-triphenyltetrazolium chloride (TTC) stain assay at $24 \mathrm{~h}$ of reperfusion after MACO surgery. The infarct volume in the Con shRNA-injected MACO mice was significantly increased compared with the NC group, but this increase was inhibited by MALAT1 shRNA injection (Fig. 5a-b).
To further verify this observation, TUNEL apoptosis detection assay was utilized. We found that TUNEL positive cells in brain infarct area slices were significantly increased in the Con shRNA-injected MACO group compared with NC group, while shMALAT1 could ameliorate cell apoptosis (Fig. 5c-d). Neurological deficit was evaluated at $24 \mathrm{~h}$ after MCAO. Neurological deficit assessment was performed by a researcher blinded to the experimental groups. Neurological function measurement was determined using Bederson scores test. The Bederson score was graded on a scale of 0 to 3 (normal score, 0; maximum score, 3), so the score of $\mathrm{NC}$ group was 0 . Consistent with the brain damage findings, the Bederson scores were significantly decreased in 
A

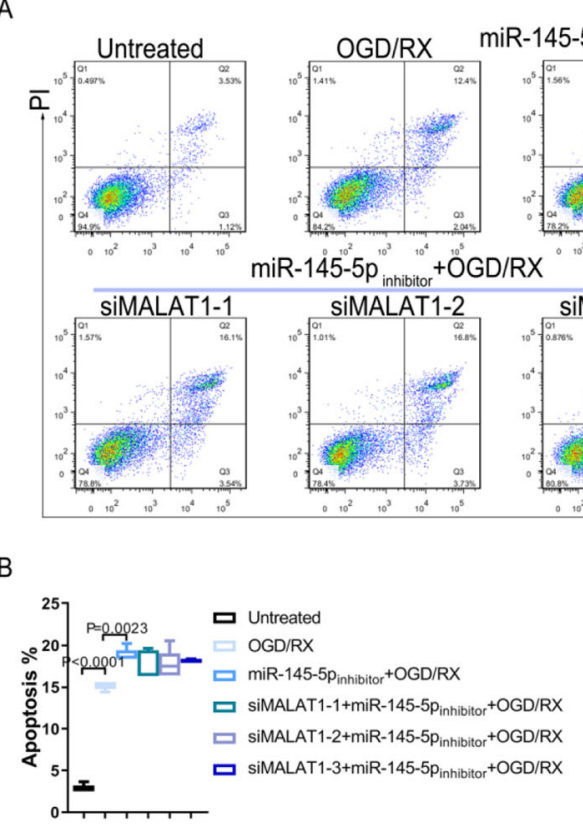

$\mathrm{C}$

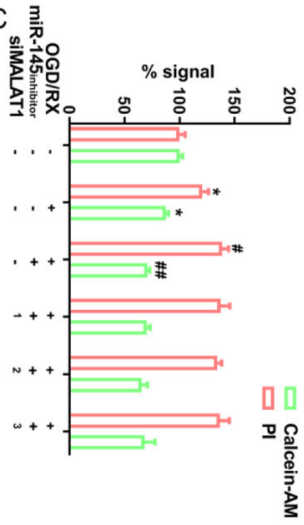

D

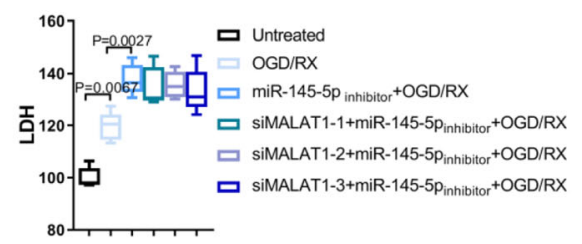

E
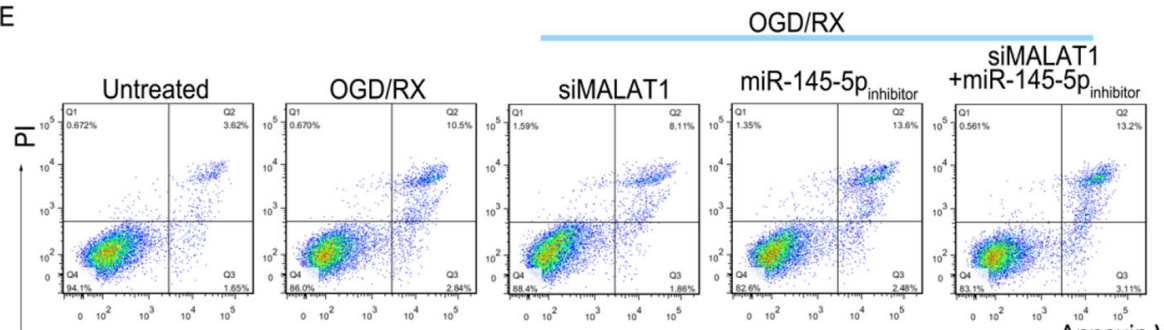

F
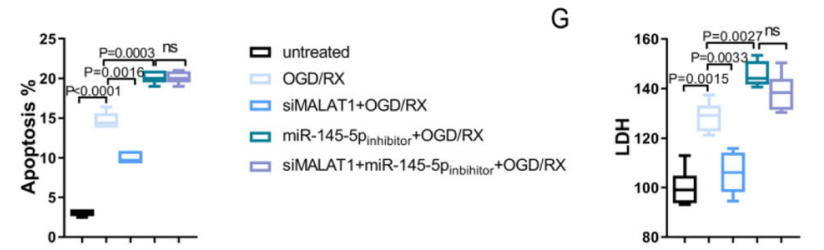

口 untreated

$\square \mathrm{OGD} / \mathrm{RX}$

D SIMALAT1+OGD/RX

口 miR-145-5 $p_{\text {inhibitor }}+O G D / R X$

siMALAT1+miR-145-5p inbihitor $+O G D / R X$

Fig. 4 MiR-145 mediates the effect of MALAT1 on the damage to astrocyte cells. a-b. Flow cytometry analysis of apoptotic cells. c. Cell CalceinAM/PI staining assay to determine cell viability. $\mathbf{d}$. LDH assay analysis to determine LDH release in different groups. e-f. Flow cytometry detection of cell apoptosis after treatment with siMALAT1 alone, miR-145 inhibitor alone, or siMALAT1 plus miR-145 inhibitor in OGD/RX conditions, and Control. g. LDH assay analysis was used to detect the LDH activity in the different groups

MALAT1 shRNA-injected mice compared with those in the Con shRNA-injected mice after $24 \mathrm{~h}$ of reperfusion (Fig. 5e).

\section{Discussion}

The development of effective treatment is critical for ischemia stroke, because it causes high mortality and disability worldwide. LncRNAs are a newly discovered class of regulators in the cerebral vascular endothelium after ischemic injury, such as stroke [29]. In recent years, non-coding RNAs have emerged as novel targets to treat ischemia stroke [30, 31]. For example, inhibition of LncCHRF reduced ischemic injury by regulating the
miR-126/SOX6 axis [32]. Knockdown of LncRNA AK038897 could protect against cerebral ischemia/reperfusion injury by regulating miR-26a-5 targeting of DAPK1 [33]. Thus, lncRNAs have great potential as new therapeutic targets in the progression of cerebral ischemia/reperfusion injury. Resent study had demonstrated that MALAT1 also play an important role in cerebral ischemia/reperfusion injury [34]. For instance, silencing of MALAT1 could inhibit OGD-R-induced apoptosis [35]. Down-regulation of MALAT1 suppressed ischemic injury and autophagy in vitro and in vivo [21]. Furthermore, MALAT1 silencing presented with larger brain infarct size, worse neurological scores, and reduced 


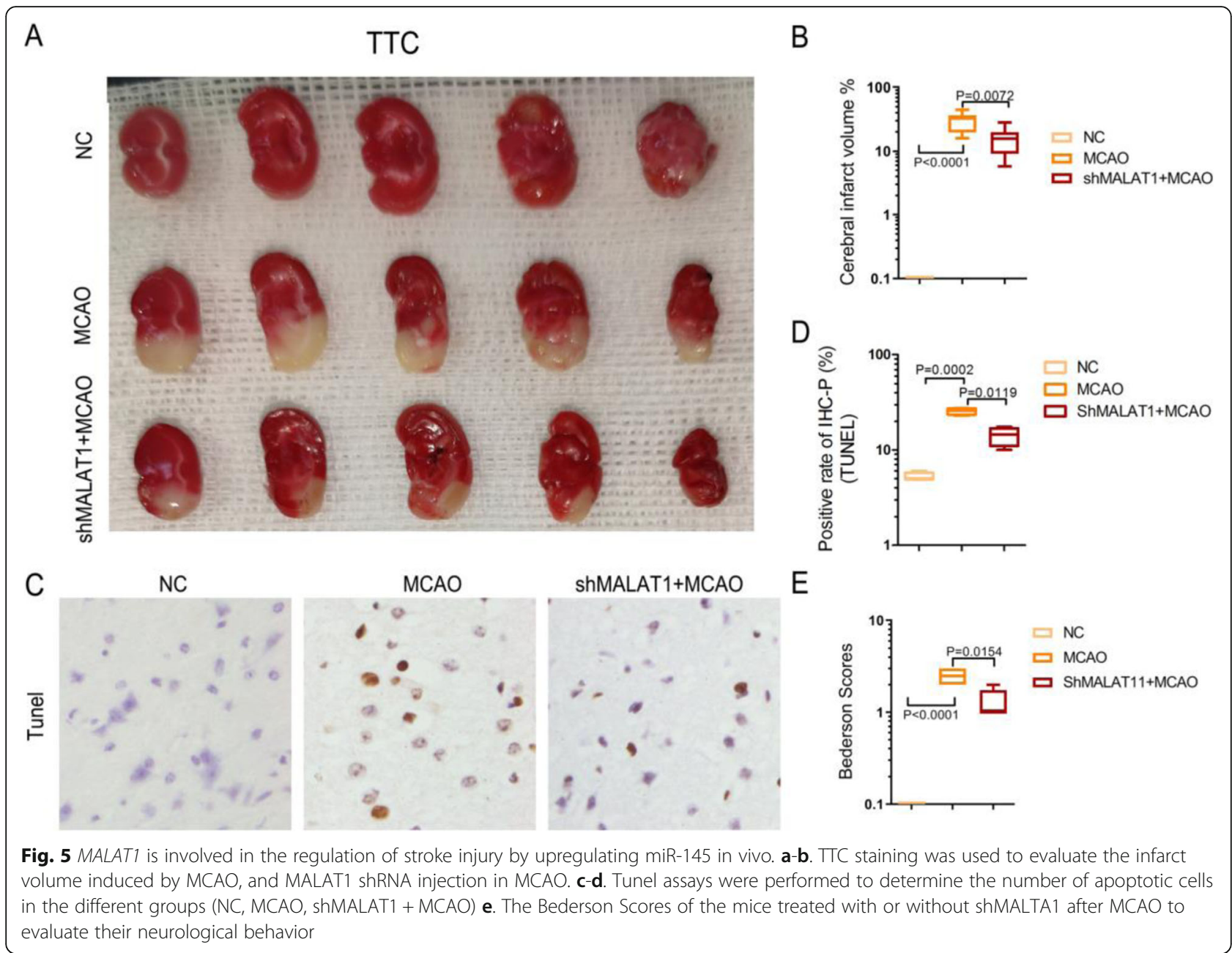

sensorimotor functions post-MCAO [20].In the present study, we demonstrated that inhibition of MALAT1 had a protective effect against cerebral ischemia/reperfusion injury by regulating miR-145/AQP4 expression. We found that MALAT1 levels were significantly upregulated in the MCAO mouse model at 24 and $48 \mathrm{~h}$ compared with sham group in vivo. Furthermore, the longer the MCAO time, the higher the MALAT1 expression. In vitro, MALAT1 expression was also significantly increased after OGD/RX in MA-C cells compared with the OGD group. These results were consistent with Guo et al reported [21]. Moreover, under OGD/RX conditions, knockdown of MALAT1 reduced cell apoptosis. Compared with the OGD/RX group, the percentage of live cells increased after transfection with the MALAT1 siRNA under OGD/RX conditions, suggesting that MALAT1 siRNA might have a protective effect in cerebral ischemia/reperfusion injury, which was consistent with the results reported in a previous study [21].

Aquaporin 4 (AQP4), a small monomer, is a hydrophobic transmembrane protein with cytosolic amino and carboxy termini. AQP4 is abundantly expressed in astrocytes and is involved in the occurrence of brain edema following intracerebral hemorrhage [36, 37]. Accumulating evidence suggests that AQP4 serves as a potential therapeutic target for ischemic injury [26, 38, 39]. In this study, we determined that the expression of AQP4 was increased after OGD/RX, and that AQP4 siRNA interference could reduce cell apoptosis, which was consistent with a previous report [26]. We also found that MALAT1 could regulate the expression of AQP4. Furthermore, transfection with the AQP4 siRNA combined with the MALAT1 siRNA under OGD/RX conditions had no significant effect on cell viability, LDH release, and apoptotic cells compared with OGD/RX alone group. These results revealed that AQP4 mediates the effect of MALAT1 on the damage to astrocytes cells.

MicroRNAs (miRNAs), short non-coding RNAs of about 18 21 nucleotides in length, can regulate mRNA translation by targeting their $3^{\prime}$ untranslated region $\left(3^{\prime}\right.$ UTR) [40]. More than $20 \%$ of miRNAs are abnormally expressed in the ischemic brain, suggesting that miRNAs 
are involved in the pathogenesis and development of ischemic stroke [41, 42]. Overexpression of miR-424 could reduce ischemic brain injury through suppressing microglia activation [43]. Increasing miR-224-3p expression could attenuate cerebral ischemia/reperfusion injury by decreasing the expression of FAK family-interacting protein (FIP200) [44]. Our previous study demonstrated that upregulation of miR-145 could reduce astrocyte injury by decreasing the expression of AQP4 [23]. The present study identified MALAT1 as a regulator of cerebral ischemia/reperfusion injury by regulating miR-145 to target AQP4. Ren et al. and Xiang et al. had indicated that MALAT1 and miR-145 could bind directly to each other, which was similar to our prediction $[45,46]$. Knockdown of MALAT1 significantly upregulated the level of miR-145, and an miR-145 inhibitor could increase the expression of AQP4. Moreover, when we transfected cells with the miR-145 inhibitor under OGD/RX condition, the astrocytes cells were damaged more seriously compared with the OGD/RX group; however, treatment with the MALAT1 siRNA abrogated the protective effect of MALAT1 following transfection with the miR-145 inhibitor under OGD/RX conditions, and the miR-145 inhibitor could reverse the protection effects of MALAT1. In vivo, we also found that inhibition of MALAT1 in the MCAO model could reduce the infarct area of the whole brain and the number of apoptotic cells.

\section{Conclusion}

In conclusion, the results of the present study demonstrated that inhibition of MALAT1 could protect against cerebral ischemia/reperfusion injury by downregulating AQP4 levels via miR-145. These findings might provide novel therapeutic targets for the treatment of cerebral ischemia stroke.

\section{Supplementary information}

Supplementary information accompanies this paper at https://doi.org/10. 1186/s12929-020-00635-0.

Additional file $\mathbf{1}$ : Figure $\mathbf{S 1}$. AQP4 could promote injury to astrocyte cells caused by ischemia reperfusion. Figure S2. A. Cell viability was determined by CCK-8 assay in different treatment groups (untreated, OGD, AQP4 plasmid+OGD, AQP4 plasmid+MALAT1 siRNA+OGD). B. MA-C cells were transfected with AQP4 plasmid, or combined with MALAT1 siRNA. OGD/RX condition was employed and the level of LDH was detected by LDH assay. C. MA-C cells were transfected with AQP4 plasmid. AQP4 protein expression were assessed using Western Blot.

\section{Abbreviations}

AQP4: Aquaporin 4; BNIP3: miR-145/BCL2 interacting protein 3; CIR: Cerebral ischemia-reperfusion; I/R: Ischemia/reperfusion; IncRNAs: Long noncoding RNAs; MALAT1: Metastasis-associated lung adenocarcinoma transcript 1; MCAO: Middle Cerebral Artery Occlusion; NEAT2: Non-coding nuclearenriched abundant transcript 2; tPA: Tissue plasminogen activator: TTC: Triphenyltetrazolium chloride

\section{Acknowledgements}

Not applicable.

\section{Authors' contributions}

Wei Chen, Ying Wu, Hongwei Wang conceived the idea; Jing Jin, Li Zheng, Ying Wu and Shufen Xie performed the experiments; Xiaoxiao Zheng, Ting Guan and Yangfan Huo analyzed the data; Wei Chen wrote the manuscript. Ying Wu revised the manuscript. All authors have read and approved the final manuscript.

\section{Authors' information}

Not applicable

\section{Funding}

This study was funded by the Zhejiang Provincial Ten Thousand Plan for Young Top Talents (2018), the Innovative Talents Training Project of Zhejiang Health Bureau (2018), the Scientific Research Project of Health and Family Planning Commission of Zhejiang Province (2016147673), Key Research \& Development Plan of Zhejiang Province (2019C03034), Health and Family planning commission of Zhejiang Province (2017KY327), the National Natural Science Foundation of China (31900543, 81972674, 81972693), the Zhejiang Provincial Nature Science Foundation of China (LR20H160001), Zhejiang Provincial Medical and Health Science and Technology Project (WKJ-ZJ-1916) and the Zhejiang Provincial Traditional Chinese Medicine Science and Technology Project (2020ZZ004)

\section{Availability of data and materials}

All data generated or analyzed during this study are included in this published article.

\section{Ethics approval and consent to participate}

All animal experiments were approved by the First Affiliated Zhejiang Hospital, Zhejiang University of Medical Ethics Committee and the Medical Faculty Ethics Committee of the First Affiliated Zhejiang Hospital, Zhejiang University in accordance with the National Institutes of Health Guide for Care and Use of Laboratory Animals (NIH Publications, No. 8023, revised 1978).

Consent for publication

Not applicable.

\section{Competing interests}

The authors declare that they have no competing interests.

\section{Author details}

'Department of anesthesiology, Tongde hospital of Zhejiang Province, Hangzhou 310012, Zhejiang, China. ${ }^{2}$ Cancer Institute of Integrated traditional Chinese and Western Medicine, Zhejiang Academy of Traditional Chinese Medicine, Tongde hospital of Zhejiang Province, NO.234, Gucui Road, Hangzhou 310012, Zhejiang, China. ${ }^{3}$ Department of Medical Oncology, Tongde hospital of Zhejiang Province, Hangzhou 310012, Zhejiang, China. ${ }^{4}$ Department of Neurosurgery, The First Affiliated Hospital, Zhejiang

University, School of Medicine, Hangzhou, Zhejiang 310003, China.

Received: 13 October 2019 Accepted: 26 February 2020

Published online: 06 March 2020

\section{References}

1. Siniscalchi A, Gallelli L, Malferrari G, Pirritano D, Serra R, Santangelo E, et al. Cerebral stroke injury: the role of cytokines and brain inflammation. J Basic Clin Physiol Pharmacol. 2014;25(2):131-7.

2. Miller JB, Merck LH, Wira CR, Meurer WJ, Schrock JW, Nomura JT, et al. The advanced reperfusion era: implications for emergency Systems of Ischemic Stroke Care. Ann Emerg Med. 2017;69(2):192-201.

3. Campbell BC, Mitchell PJ, Kleinig TJ, Dewey HM, Churilov L, Yassi N, et al. Endovascular therapy for ischemic stroke with perfusion-imaging selection. N Engl J Med. 2015;372(11):1009-18.

4. Jean WC, Spellman SR, Nussbaum ES, Low WC. Reperfusion injury after focal cerebral ischemia: the role of inflammation and the therapeutic horizon. Neurosurgery. 1998:43(6):1382-96 discussion 96-7.

5. Mehta SL, Manhas N, Raghubir R. Molecular targets in cerebral ischemia for developing novel therapeutics. Brain Res Rev. 2007;54(1):34-66. 
6. Fan J, Liu Y, Yin J, Li Q, Li Y, Gu J, et al. Oxygen-glucose-deprivation/ Reoxygenation-induced Autophagic cell death depends on JNK-mediated phosphorylation of Bcl-2. Cell Physiol Biochem. 2016;38(3):1063-74.

7. Blakeley JO, Llinas RH. Thrombolytic therapy for acute ischemic stroke. J Neurol Sci. 2007;261(1-2):55-62.

8. Yang F, Bi J, Xue X, Zheng L, Zhi K, Hua J, et al. Up-regulated long noncoding RNA H19 contributes to proliferation of gastric cancer cells. FEBS J. 2012;279(17):3159-65.

9. Geisler S, Coller J. RNA in unexpected places: long non-coding RNA functions in diverse cellular contexts. Nat Rev Mol Cell Biol. 2013;14(11): 699-712.

10. Martianov I, Ramadass A, Serra Barros A, Chow N, Akoulitchev A. Repression of the human dihydrofolate reductase gene by a non-coding interfering transcript. Nature. 2007;445(7128):666-70.

11. Mercer TR, Mattick JS. Structure and function of long noncoding RNAs in epigenetic regulation. Nat Struct Mol Biol. 2013;20(3):300-7.

12. Yin KJ, Hamblin M, Chen YE. Non-coding RNAs in cerebral endothelia pathophysiology: emerging roles in stroke. Neurochem Int. 2014;77:9-16.

13. Li C, Chen J, Zhang K, Feng B, Wang R, Chen L. Progress and prospects of Long noncoding RNAs (IncRNAs) in hepatocellular carcinoma. Cell Physiol Biochem. 2015:36(2):423-34.

14. Gutschner T, Hammerle M, Eissmann M, Hsu J, Kim Y, Hung G, et al. The noncoding RNA MALAT1 is a critical regulator of the metastasis phenotype of lung cancer cells. Cancer Res. 2013;73(3):1180-9.

15. Gutschner T, Hammerle M, Diederichs S. MALAT1 -- a paradigm for long noncoding RNA function in cancer. J Mol Med (Berl). 2013;91(7):791-801.

16. Yu Q, Xiang L, Chen Z, Liu X, Ou H, Zhou J, et al. MALAT1 functions as a competing endogenous RNA to regulate SMAD5 expression by acting as a sponge for miR-142-3p in hepatocellular carcinoma. Cell Biosci. 2019;9:39.

17. Tang D, Yang Z, Long F, Luo L, Yang B, Zhu R, et al. Long noncoding RNA MALAT1 mediates stem cell-like properties in human colorectal cancer cells by regulating miR-20b-5p/Oct4 axis. J Cell Physiol. 2019;234(11):20816-828.

18. Michalik KM, You X, Manavski Y, Doddaballapur A, Zornig M, Braun T, et al. Long noncoding RNA MALAT1 regulates endothelial cell function and vessel growth. Circ Res. 2014;114(9):1389-97.

19. Zhang J, Yuan L, Zhang X, Hamblin MH, Zhu T, Meng F, et al. Altered long non-coding RNA transcriptomic profiles in brain microvascular endothelium after cerebral ischemia. Exp Neurol. 2016;277:162-70.

20. Zhang $X$, Tang $X$, Liu K, Hamblin MH, Yin KJ. Long noncoding RNA Malat1 regulates cerebrovascular pathologies in ischemic stroke. J Neurosci. 2017; 37(7):1797-806.

21. Guo D, Ma J, Yan L, Li T, Li Z, Han X, et al. Down-regulation of Lncrna MALAT1 attenuates neuronal cell death through suppressing Beclin1dependent autophagy by regulating Mir-30a in cerebral ischemic stroke. Cell Physiol Biochem. 2017:43(1):182-94

22. Zhao ZH, Hao W, Meng QT, Du XB, Lei SQ, Xia ZY. Long non-coding RNA MALAT1 functions as a mediator in cardioprotective effects of fentanyl in myocardial ischemia-reperfusion injury. Cell Biol Int. 2017;41(1):62-70.

23. Zheng $L$, Cheng W, Wang X, Yang Z, Zhou X, Pan C. Overexpression of MicroRNA-145 ameliorates astrocyte injury by targeting aquaporin 4 in cerebral ischemic stroke. Biomed Res Int. 2017;2017:9530951.

24. Iwata-Ichikawa E, Kondo Y, Miyazaki I, Asanuma M, Ogawa N. Glial cells protect neurons against oxidative stress via transcriptional up-regulation of the glutathione synthesis. J Neurochem. 1999;72(6):2334-44.

25. Lin SP, Ye S, Long Y, Fan Y, Mao HF, Chen MT, et al. Circular RNA expression alterations are involved in OGD/R-induced neuron injury. Biochem Biophys Res Commun. 2016;471(1):52-6.

26. Zheng $Y$, Pan C, Chen M, Pei A, Xie L, Zhu S. miR29a ameliorates ischemic injury of astrocytes in vitro by targeting the water channel protein aquaporin 4. Oncol Rep. 2019;41(3):1707-17.

27. Livak KJ, Schmittgen TD. Analysis of relative gene expression data using real-time quantitative $P C R$ and the 2(-Delta Delta $C(T))$ method. Methods. 2001;25(4):402-8

28. Zhong Y, Yu C, Qin W. LncRNA SNHG14 promotes inflammatory response induced by cerebral ischemia/reperfusion injury through regulating miR136-5p /ROCK1. Cancer Gene Ther. 2019;26(7-8):234-47.

29. Zhang Q, Matsuura K, Kleiner DE, Zamboni F, Alter HJ, Farci P. Analysis of long noncoding RNA expression in hepatocellular carcinoma of different viral etiology. J Transl Med. 2016;14(1):328.

30. Bao MH, Szeto V, Yang BB, Zhu SZ, Sun HS, Feng ZP. Long non-coding RNAs in ischemic stroke. Cell Death Dis. 2018;9(3):281.
31. Chandran R, Mehta SL, Vemuganti R. Non-coding RNAs and neuroprotection after acute CNS injuries. Neurochem Int. 2017;111:12-22.

32. Gai HY, Wu C, Zhang Y, Wang D. Long non-coding RNA CHRF modulates the progression of cerebral ischemia/reperfusion injury via miR-126/SOX6 signaling pathway. Biochem Biophys Res Commun. 2019;514(2):550-7.

33. Wei R, Zhang L, Hu W, Wu J, Zhang W. Long non-coding RNA AK038897 aggravates cerebral ischemia/reperfusion injury via acting as a ceRNA for miR-26a-5p to target DAPK1. Exp Neurol. 2019;314:100-10.

34. Alishahi M, Ghaedrahmati F, Kolagar TA, Winlow W, Nikkar N, Farzaneh M, et al. Long non-coding RNAs and cell death following ischemic stroke. Metab Brain Dis. 2019;34(5):1243-51.

35. Xin JW, Jiang YG. Long noncoding RNA MALAT1 inhibits apoptosis induced by oxygen-glucose deprivation and reoxygenation in human brain microvascular endothelial cells. Exp Ther Med. 2017;13(4):1225-34.

36. Fu X, Li Q, Feng Z, Mu D. The roles of aquaporin-4 in brain edema following neonatal hypoxia ischemia and reoxygenation in a cultured rat astrocyte model. Glia. 2007:55(9):935-41.

37. Xu J, Qiu GP, Huang J, Zhang B, Sun SQ, Gan SW, et al. Internalization of aquaporin-4 after collagenase-induced intracerebral hemorrhage. Anat Rec (Hoboken). 2015;298(3):554-61.

38. Zheng $Y$, Wang $L$, Chen M, Pei A, Xie L, Zhu S. Upregulation of miR-130b protects against cerebral ischemic injury by targeting water channel protein aquaporin 4 (AQP4). Am J Transl Res. 2017;9(7):3452-61.

39. Mokhtarudin MJ, Payne SJ. The study of the function of AQP4 in cerebral ischaemia-reperfusion injury using poroelastic theory. Int J Numer Method Biomed Eng. 2017;33(1).

40. Bushati N, Cohen SM. microRNA functions. Annu Rev Cell Dev Biol. 2007;23: 175-205.

41. Rink C, Khanna S. MicroRNA in ischemic stroke etiology and pathology. Physiol Genomics. 2011;43(10):521-8.

42. Tan JR, Tan KS, Koo YX, Yong FL, Wang CW, Armugam A, et al. Blood microRNAs in low or no risk ischemic stroke patients. Int J Mol Sci. 2013; 14(1):2072-84.

43. Zhao H, Wang J, Gao L, Wang R, Liu X, Gao Z, et al. MiRNA-424 protects against permanent focal cerebral ischemia injury in mice involving suppressing microglia activation. Stroke. 2013;44(6):1706-13.

44. Deng Y, Ma G, Dong Q, Sun X, Liu L, Miao Z, et al. Overexpression of miR224-3p alleviates apoptosis from cerebral ischemia reperfusion injury by targeting FIP200. J Cell Biochem. 2019:120(10):17151-158.

45. Ren L, Wei C, Li K, Lu Z. LncRNA MALAT1 up-regulates VEGF-A and ANGPT2 to promote angiogenesis in brain microvascular endothelial cells against oxygen-glucose deprivation via targetting miR-145. Biosci Rep. 2019;39(3).

46. Xiang Y, Zhang Y, Tang Y, Li Q. MALAT1 modulates TGF-beta1-induced endothelial-to-Mesenchymal transition through Downregulation of miR-145. Cell Physiol Biochem. 2017;42(1):357-72.

\section{Publisher's Note}

Springer Nature remains neutral with regard to jurisdictional claims in published maps and institutional affiliations.

Ready to submit your research? Choose BMC and benefit from:

- fast, convenient online submission

- thorough peer review by experienced researchers in your field

- rapid publication on acceptance

- support for research data, including large and complex data types

- gold Open Access which fosters wider collaboration and increased citations

- maximum visibility for your research: over $100 \mathrm{M}$ website views per year

At $\mathrm{BMC}$, research is always in progress.

Learn more biomedcentral.com/submission 